\title{
Comparison of timing jitter in external and monolithic cavity mode-locked semiconductor lasers
}

\author{
D. J. Derickson, P. A. Morton, and J. E. Bowers \\ ECE Department, University of California, Santa Barbara, California 93106 \\ R. L. Thornton \\ Xerox Research Center, Palo Alto, California 94304
}

(Received 22 May 1991; accepted for publication 26 September 1991)

\begin{abstract}
A comprehensive timing jitter comparison is made for mode-locked semiconductor lasers using active, passive, and hybrid mode-locking techniques in both external and monolithic cavity configurations. Active mode locking gives the lowest residual rms timing jitter of $65 \mathrm{fs}$ ( $150 \mathrm{~Hz}-50 \mathrm{MHz}$ ), followed by the hybrid and passive mode-locking techniques. It is found that monolithic cavity devices with all active waveguides have higher timing jitter levels than the comparable external cavity case.
\end{abstract}

Mode-locked semiconductor lasers have produced ultrashort optical pulses in monolithic ${ }^{1-4}$ and external cavity configurations. ${ }^{5}$ Monolithic cavities offer the advantage of mechanical stability, small size, and ease of use as compared to external cavity devices. In this letter, monolithic and external cavity multiquantum well lasers are compared using active, passive, and hybrid mode-locking techniques. Quantum well rather than bulk active region lasers are chosen because of the larger ratio in the differential gain between the reverse biased saturable absorber segments and the forward biased gain segments. ${ }^{6}$ In addition to pulse-width and spectral-width measurements, this paper concentrates on a comparison of the timing jitter properties of the lasers. Pulse to pulse timing jitter is an important noise parameter that contributes directly to the time resolution in most applications of mode-locked lasers.

Figure 1 shows a diagram of the monolithic and external cavity devices used in the active, passive, and hybrid mode-locking experiments. The active region for all of the devices is comprised of four GaAs quantum wells with the lateral index guide formed by impurity induced disordering. ${ }^{7}$ The monolithic structure is $6.1-\mathrm{mm}$ long with the top electrode divided into two short end segments and a long center section. With all sections connected together, the device has a threshold of $115 \mathrm{~mA}$ and a single facet differential quantum efficiency of $4 \%$. The $80-\mu \mathrm{m}$ end segment is reverse biased for use as a saturable absorber, terminated in $50 \Omega$, and high-reflection coated. The impulse response of the saturable absorber was measured using a sampling oscilloscope as the termination. The loss recovers quickly ( 50 ps full width at half-maximum). The middle section is dc biased to act as an active waveguide. The $400-\mu \mathrm{m}$ end segment is modulated with a $24.5-\mathrm{dBm}, 5.5-$ $\mathrm{GHz}$ sinusoid. The external cavity device of Fig. 1(b) is a two segment device cleaved from one of the monolithic devices. The external cavity laser has a threshold current of $13 \mathrm{~mA}$ with an output facet differential quantum efficiency of $10 \%$. The absorber facet has a $70 \%$ reflection coating and the opposite facet is antireflection coated for external cavity operation at a $5.5-\mathrm{GHz}$ repetition rate.

Table I gives a summary of the bias conditions and the performance results for active, passive, and hybrid mode locking of the monolithic and external cavity configurations. The average output power is held at $1 \mathrm{~mW}$ in all cases and the mode-locking frequency is nominally 5.5 $\mathrm{GHz}$. The modulation frequency in the active and hybrid mode-locking cases is adjusted to give the shortest optical pulse widths compatible with low amplitude noise and timing jitter. It is found that modulation frequencies slightly lower than those for minimum pulse width give the most stable results with higher modulation frequencies producing amplitude and timing instabilities. ${ }^{8}$ In all cases the pulses have a large time bandwidth product and frequency chirp. The excess bandwidth is due to self-phase modulation of the pulses as the carrier density and index of refraction change during the pulse propagation. ${ }^{9}$ Hybrid mode locking produces the shortest pulse widths of 6.5 and $2.5 \mathrm{ps}$, in the monolithic and external cavity cases, respectively, due to the combined action of saturable absorption, saturable gain, and active gain modulation.

The absolute and residual timing jitter performance of the devices was compared using the frequency domain techniques discussed in Ref. 10. Absolute timing jitter is due to contributions from the modulation source and the mode-locked laser, whereas residual timing jitter is that due to the mode-locked laser only. Passive mode-locking

TABLE I. Bias and performance comparison.

\begin{tabular}{|c|c|c|c|c|c|c|}
\hline $\begin{array}{l}\text { Mode- } \\
\text { locking } \\
\text { technique }\end{array}$ & $\begin{array}{c}\text { Saturable } \\
\text { absorber } \\
\text { bias } \\
\text { I a or V }\end{array}$ & $\begin{array}{c}\text { Center } \\
\text { section } \\
\text { bias } \\
\text { I } \mathrm{c}(\mathrm{mA})\end{array}$ & $\begin{array}{c}\text { End } \\
\text { section } \\
\text { bias } \\
\text { I e, Prf }\end{array}$ & $\begin{array}{c}\text { Pulse } \\
\text { width } \\
\text { (ps) }\end{array}$ & $\begin{array}{c}\text { Spectral } \\
\text { width } \\
\text { (GHz) }\end{array}$ & $\begin{array}{c}\text { Time/ } \\
\text { band- } \\
\text { width } \\
\text { product }\end{array}$ \\
\hline $\begin{array}{l}\text { Active } \\
\text { monolithic } \\
\text { Passive }\end{array}$ & $2.0 \mathrm{~mA}$ & 126 & $\begin{array}{l}12 \mathrm{~mA} \\
24.5 \mathrm{dBm}\end{array}$ & 13 & 330 & 4.3 \\
\hline $\begin{array}{l}\text { monolithic } \\
\text { Hybrid }\end{array}$ & $\ldots .1 \mathrm{~V}$ & 170 & $\begin{array}{l}10 \mathrm{~mA} \\
10 \mathrm{~mA}\end{array}$ & 10 & 400 & 4.0 \\
\hline $\begin{array}{l}\text { monolithic } \\
\text { Active }\end{array}$ & $-1 \mathrm{~V}$ & 160 & $\begin{array}{l}24.5 \mathrm{dBm} \\
18 \mathrm{~mA}\end{array}$ & 6.5 & 540 & 3.5 \\
\hline $\begin{array}{l}\text { external } \\
\text { Passive }\end{array}$ & $2.0 \mathrm{~mA}$ & $\cdots$ & $24.5 \mathrm{dBm}$ & 9 & 320 & 2.9 \\
\hline $\begin{array}{l}\text { external } \\
\text { Hybrid }\end{array}$ & $-1 \mathrm{~V}$ & $\cdots$ & $\begin{array}{l}80 \mathrm{~mA} \\
80 \mathrm{~mA}\end{array}$ & 2.5 & 720 & 1.8 \\
\hline external & $-1 V$ & $\cdots$ & $24.5 \mathrm{dBm}$ & 2.7 & 800 & 2.2 \\
\hline
\end{tabular}




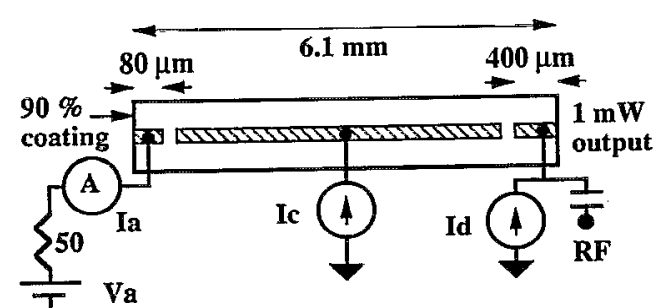

(a)

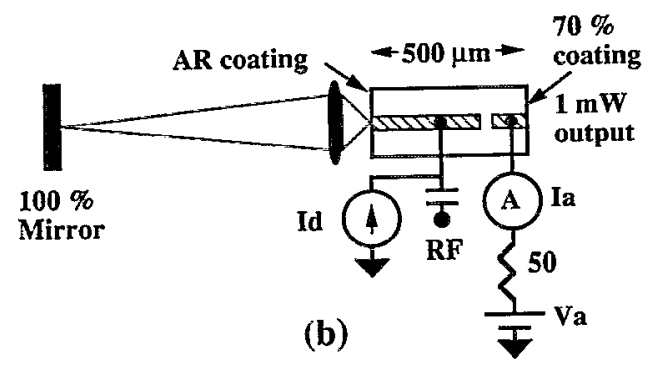

FIG. 1. (a) Three section monolithic cavity mode-locked laser used in the experiment. (b) Two section external cavity mode-locked laser used in the experiment.

only has absolute timing jitter since no modulation source is involved. The single sideband phase noise level relative to the carrier per $1-\mathrm{Hz}$ bandwidth, $\mathscr{L}(f)$, is measured in these experiments. $\mathscr{L}(f)$ can be converted to a time domain figure of merit called the root-mean-square (rms) timing jitter by using the following expression:

$$
\sigma_{\mathrm{rms}}=\frac{1}{2 \pi n f_{\text {mod }}} \sqrt{2 \int_{f_{\mathrm{low}}}^{f_{\text {high }}} \mathscr{L}(f) d f}
$$

where $\sigma_{\mathrm{rms}}$ is the rms timing jitter, $n$ is the harmonic number, $f_{\text {mod }}$ is the modulation frequency, and $f_{\text {low }}$ and $f_{\text {high }}$ are limits to the integration with respect to the offset frequency.

Figure 2 shows absolute $\mathscr{L}(f)$ for the active external and active monolithic cavity mode-locked lasers for the first three harmonics of the $5.5-\mathrm{GHz}$ mode-locking frequency. Amplitude noise can be distinguished from phase

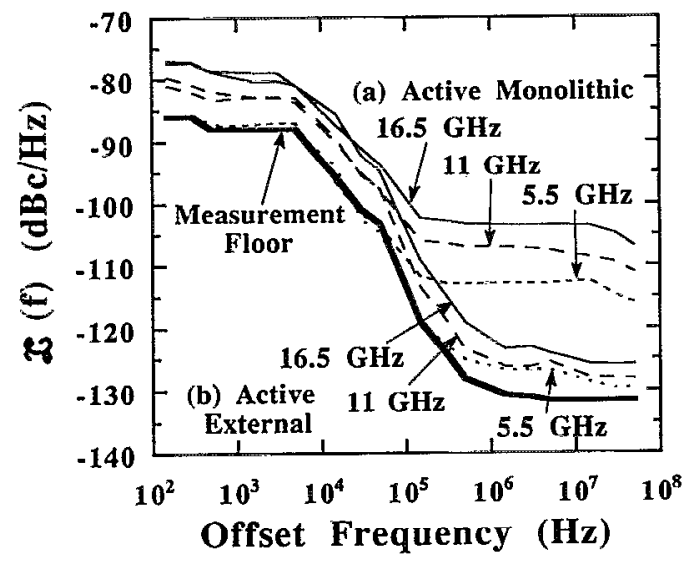

FIG. 2. The absolute noise from (a) the actively mode-locked monolithic cavity device and (b) the actively mode-locked external cavity device. The measurement fioor represents the noise contribution from the modulation source and the local oscillator of the spectrum analyzer. noise by noting how the relative noise level changes with harmonic number for a constant offset frequency. The relative phase noise level will increase by 6 and $9.5 \mathrm{~dB}$, respectively, for the second and third harmonic, whereas the relative amplitude noise level will remain constant with harmonic number. The floor curve shows the limitation of the measurement system due to the spectral purity of the modulation source and the local oscillator in the spectrum analyzer. The noise at offsets below $100 \mathrm{kHz}$ is phase noise in nature and is dominated by the modulating source. Beyond $100 \mathrm{kHz}$, the noise from the monolithic cavity device becomes dominant and is phase noise in nature as is shown by the rising noise level with harmonic number. The absolute timing jitter for this case is $600 \mathrm{fs}(150 \mathrm{~Hz}-50 \mathrm{MHz})$, with the dominant source of the jitter being the modelocked laser. Figure 2 shows that the actively mode-locked external cavity laser starts to add noise at offsets larger than $300 \mathrm{kHz}$. This contribution is amplitude noise because its relative level remains constant with harmonic number. The absolute timing jitter in this case is $<240 \mathrm{fs}$ and is dominated by the modulation source. Figure 2 illustrates that monolithic cavity mode-locked lasers with all-active wavcguides have significantly higher jitter than their external cavity counterparts. Timing jitter introduced by actively and hybridly mode-locked lasers is partially a result of index of refraction fluctuations caused by carrier density fluctuations which are dominated by spontaneous emission noise. The idea that the index of refraction fluctuations modulate the round trip time of the laser is one possible explanation and clearly more theoretical work remains to be done.

Figure 3 compares the residual phase noise of the hybrid and active mode-locked devices and the absolute noise of the passive mode-locked devices. This plot compares the noise contribution of the laser only, independent of modulation sources. Table II lists the timing jitter results and intensity noise levels for the six experiments. The actively

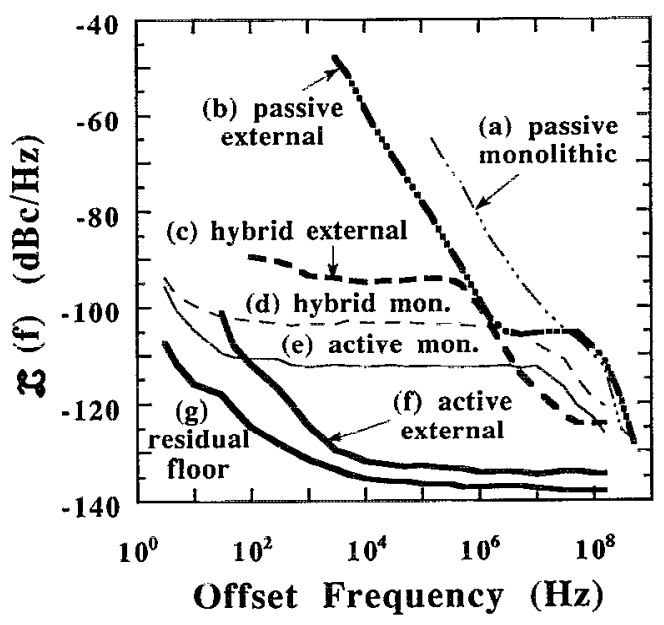

FIG. 3. The absolute timing jitter for the (a) passive monolithic and (b) passive external mode-locking experiments. The residual timing jitter for the (c) hybrid external, (d) hybrid monolithic, (e) active monolithic, and (f) active external mode-locked laser experiments. The residual noise floor $(\mathrm{g})$ is measured by bypassing the mode-locked laser. 
TABLE II. Comparison of timing jitter and intensity noise levels.

\begin{tabular}{|c|c|c|c|}
\hline $\begin{array}{l}\text { Mode- } \\
\text { locking } \\
\text { technique }\end{array}$ & $\begin{array}{c}\text { Absolute rms } \\
\text { timing jitter } \\
150 \mathrm{~Hz}-50 \mathrm{MHz}\end{array}$ & $\begin{array}{c}\text { Residual rms } \\
\text { timing jitter } \\
150 \mathrm{~Hz}-50 \mathrm{MHz}\end{array}$ & $\begin{array}{c}\text { Relative } \\
\text { intensity noise } \\
\text { at } 100 \mathrm{MHz}\end{array}$ \\
\hline Active monolithic & $600 \mathrm{fs}$ & $530 \mathrm{fs}$ & $-122 \mathrm{~dB} / \mathrm{Hz}$ \\
\hline Passive monolithic & $\begin{array}{c}12.5 \mathrm{ps} \\
(150 \mathrm{kHz}-50 \mathrm{MHz})\end{array}$ & $\begin{array}{c}12.5 \mathrm{ps} \\
(150 \mathrm{kHz}-50 \mathrm{MHz})\end{array}$ & $116 \mathrm{~dB} / \mathrm{Hz}$ \\
\hline Hybrid monolithic & $1200 \mathrm{fs}$ & $1130 \mathrm{fs}$ & $-109 \mathrm{~dB} / \mathrm{Hz}$ \\
\hline Active external & $240 \mathrm{fs}$ & $65 \mathrm{fs}$ & $<-126 \mathrm{~dB} / \mathrm{Hz}$ \\
\hline Passive external & $\begin{array}{c}12.2 \mathrm{ps} \\
(1.5 \mathrm{kHz}-50 \mathrm{MHz})\end{array}$ & $\begin{array}{c}12.2 \mathrm{ps} \\
(1.5 \mathrm{kHz}-50 \mathrm{MHz})\end{array}$ & $-103 \mathrm{~dB} / \mathrm{Hz}$ \\
\hline Hybrid external & $1060 \mathrm{fs}$ & $980 \mathrm{fs}$ & $-105 \mathrm{~dB} / \mathrm{Hz}$ \\
\hline
\end{tabular}

mode-locked monolithic and external cavity devices differ in the length and carrier density of the active waveguide in the cavity. Carrier density fluctuations modulate the round trip delay time for the optical pulses. The delay variations for the monolithic cavity device are larger because of the longer active waveguide length in the cavity. For similar carrier density levels in each active waveguide, the phase modulation level will be larger in the monolithic cavity device than in the external cavity device by the ratio of the active waveguide lengths. $\mathscr{L}(f)$ would therefore increase by the active waveguide lengths squared. For active waveguide lengths of 6.1 and $0.5 \mathrm{~mm}$ in the monolithic and external cavity devices, respectively, this corresponds to a 22-dB increase. This compares well with the measured 23$\mathrm{dB}$ increase at offset frequencies between $5 \mathrm{kHz}$ and 50 MHz. Noise with $1 / f$ slope is observed in the output of the mode-locked lasers at low offset frequencies and the noise contribution from the laser falls off above the relaxation resonance frequencies.

Passively mode-locked lasers have the highest timing jitter levels due to the absence of a high stability driving source. A conventional oscillator has the proportionality ${ }^{11}$

$$
\mathscr{L}(f) \propto \frac{P_{\text {noise }} f_{\text {cavity }}^{2}}{Q^{2} f^{2}} .
$$

$P_{\text {noise }}$ is the noise power, $f_{\text {cavity }}$ is the cavity resonance frequency, $f$ is the offset frequency, and $Q$ is the quality factor. This indicates that $\mathscr{L}(f)$ drops off at a $20-\mathrm{dB} /$ decade rate as is seen in Fig. 3. The timing jitter of the monolithic cavity device is larger than that of the external cavity device partially due to the lower device $Q$ of the monolithic device. The lower $Q$ of the monolithic cavity device is due to the dominance of the $7.5 / \mathrm{cm}$ waveguide loss for the $0.61-\mathrm{cm}-$ long device. Because of the $20-\mathrm{dB} /$ decade $\mathscr{L}(f)$ rolloff, the timing jitter will be dominated by the phase noise at low offset frequencies.

For either the monolithic or external cavity case, Fig. 3 shows that hybrid mode locking gives a higher noise level than active mode locking. The residual timing jitter of the hybridally mode-locked external and monolithic cavity devices is 980 and $1100 \mathrm{fs}$, respectively, and is laser dominated. When the absorber section is reverse-biased, the gain segment carrier density level must be substantially increased to overcome the loss of the saturable absorber.
This carrier density rise is especially high in the external cavity case where all of the extra gain must be made up in a relatively short gain length. For the external case, the pumping current was increased from 18 to $80 \mathrm{~mA}$. The higher carrier density level and decreased carrier recombination lifetimes cause increased spontaneous emission levels in the external cavity deviccs but the shorter active waveguide length reduces the contribution to phase noise and timing jitter.

In summary, it is shown that the monolithic cavity devices with active waveguides have larger timing jitter compared to external cavity devices of equivalent length. For either monolithic or external cavity devices, active mode locking gives the lowest jitter level, followed by hybrid and passive mode locking. One possible explanation for the larger timing jitter in monolithic cavity devices is due to index of refraction variations occuring along the entire cavity length compared to a small fraction of the cavity length in the external cavity case. Passively modelocked lasers have the largest timing jitter levels due to the fact that they are free running oscillators with relatively low device $Q$.

This work was sponsored by the Office of Naval Research. The authors would like to acknowledge contributions from Roger Helkey, Paul Humphrey, Gang Jia, Alan Mar, and Tom Reynolds.

'Y. K. Chen, M. C. Wu, T. Tanbun-Ek, R. A. Logan, and M. A. Chin, Appl. Phys. Lett. 58, 1253 (1991).

${ }^{2}$ R. S. Tucker, U. Koren, G. Raybon, C. A. Burrus, B. I. Miller, T. L.Koch, G. Eisenstein, and A. Shahar, Electron. Lett. 25, 622 (1989).

${ }^{3}$ S. Sanders, A. Yariv, J. Paslaski, J. Ungar, and H. Zarem, Appl. Phys. Lett. 58, 681 (1991).

${ }^{4}$ P. A. Morton, J. E. Bowers, L. A. Koszi, M. Soler, J. Lopata, and D. P. Wilt, Appl. Phys. Lett. 56, 111 (1990).

${ }^{5}$ J. E. Bowers, P. A. Morton, A. Mar, and S. W. Corzine, IEEE J. Quantum Electron. QE-25, 1426 (1989).

${ }^{6}$ K. Y. Lau, IEEE J. Quantum Electron. QE-26, 250 (1990).

${ }^{7}$ R. L. Thornton, W. J. Mosby, and T. J. Paoli, IEEE J. Lightwave Technol. 6, 786 (1988).

${ }^{8}$ A. J. Lowry, IEE Proc. J. 136, 264 (1989).

${ }^{9}$ G. P. Agrawal and N. A. Olsson, IEEE I. Quantum Electron. QE-25, 2297 (1989).

${ }^{10}$ D. J. Derickson, A. Mar, and J. E. Bowers, Electron. Lett. 26, 2026 (1990).

"W. P. Robbins, Phase Noise in Signal Sources, IEE Telecommunications Series 9 (Peter Peregrinus, London, 1984), p. 53. 